\title{
Wound Complications Due to Obesity in the Patients Undergoing Abdominal Surgery
}

\author{
Ammar Rasool, ${ }^{1}$ Yar Muhammad, ${ }^{2}$ Khalid Masood Gondal, ${ }^{3}$ Umair Ahmed Khan ${ }^{4}$
}

\begin{abstract}
Various factors may affect the outcome of patients in perioperative period. Obesity is considered as one of these factors which increases the risk of perioperative complications. The wound complications in obese and non-obese are different.

Objective: The objective of the study was to compare wound complications in obese and non-obese patients undergoing abdominal surgery.
\end{abstract}

Methods: This comparative study was conducted from 01- 05-2012 to 30-04-2015 on 140 patients in the department of surgery, Mayo Hospital Lahore. All these patients were divided into two groups of 70 patients each. Group A (obese) and B (nonobese) on the basis of body mass index. The patients of age less than 12 years, peritonitis, diabetes mellitus, jaundice, renal failure, coagulation disorder were excluded in both groups. All the patients had open and laparoscopic surgery by the consultant on elective list. The operative

\footnotetext{
${ }^{1}$ Medical Officer/PG Trainee, Dept of Surgery, Mayo Hospital, Lahore.

${ }^{2}$ Assistant Professor, Dept of Surgery, KEMU/Mayo Hospital, Lahore.

${ }^{3}$ Chairman, Dept of Surgery, KEMU/Mayo Hospital, Lahore.

${ }^{4}$ Medical Officer/PG Trainee, Dept of Surgery, Mayo Hospital, Lahore.
}

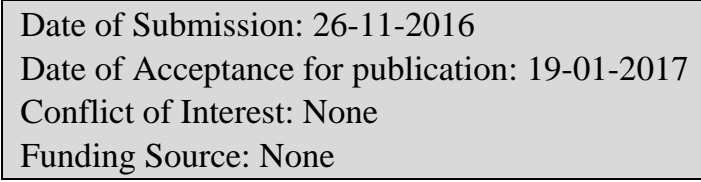

\section{Contribution}

All Authors have contributed in Study Design, Data Collection, Data Analysis, Data Interpretation, Manuscript Writing and Approval. and postoperative findings and outcome in term of wound complications like wound infection, dehiscence, seroma, hernia, were noted.

Results: All the140patients were divided into group A (obese) and B (non obese) of 70 patients each. The age ranged from 17 to 58 years and males were common $(50.8 \%)$ than females $(49.2 \%)$. In group A, body mass index varied from $31.9 \mathrm{~kg} \mathrm{~m}^{-2}$ to $39.08 \mathrm{~kg} \mathrm{~m}^{-2}$ and in group B from $19.3 \mathrm{~kg} \mathrm{~m}^{-2}$ to $23.2 \mathrm{~kg} \mathrm{~m}^{-2}$. The complications in group A were wound infection in 18, dehiscence in 5 , seroma in 13 , and hernia 4 patients. While in group B, the complications were wound infection in 3 , seroma in 5 , no dehiscence and incisional hernia in one patient. The results showed that early and late wound complications are more in obese patients.

Conclusion: The wound complications are significantly associated with obesity in patients undergoing abdominal surgery and are more in obese than in non obese patients.

Key words: Obese, Wound, Complications, Abdominal Surgery.

\section{Introduction}

Obesity is one of the comorbid conditions which can affect the operative and postoperative outcome of patients undergoing surgery. ${ }^{1}$ It is better expressed by body mass index (BMI). BMI is calculated from weight and height. Body mass index of less than 18.5 is underweight, 18.5 to 24.9 is normal, 25 to 29.9 is overweight and more than 30 is obesity. ${ }^{2}$ The distribution of body fat between visceral and non visceral compartments and within different subcutaneous areas is important clinically. Both the central and peripheral types of obesity are associated with surgical complications. $^{3,4}$ 
The abdominal surgery may be open or laparoscopic and elective or emergency. Possible complications of abdominal surgery may be local or systemic. ${ }^{5}$ The local complications include haemorrhage, infection, damage to internal organs, formation of adhesions, bowel obstruction, seroma, wound dehiscence, hernia. ${ }^{6}$ The systemic complications are like chest infection, septicaemia, deep venous thrombosis, nosocomial infections, ischaemic heart disease and some procedure related complication. ${ }^{7}$ Infectious complications of abdominal surgery range from skin and soft tissue infection to intra-abdominal abscess and peritonitis. It can be due type of surgery, diabetes mellitus, poor technique, obesity or immunopathy. ${ }^{8}$ Seroma can be due to subcutaneous potential space or inadequate drainage. This may lead to wound infection. ${ }^{9}$ Abdominal wound dehiscence, in particular, leads to incisional hernia. Risk factors for the development of wound dehiscence and hernia include chronic pulmonary disease, ascites, jaundice, anaemia, obesity, coughing, type of surgery, wound infection and closure technique .Incisional hernias can occur after any type of abdominal wall incision but midline incisions are more prone to have this complication. ${ }^{10}$

Perioperative risk factors are age, hypertension, diabetes mellitus, heart diseases, renal failure, cirrhosis, pulmonary disease, endocrine disorders, smoking, obesity and coagulopathy. These disorders need to be controlled before the surgery. The operative problems associated with obesity are difficulty in exposure and closure, port insertion, bleeding, prolonged operative time, visceral damage, wound infection, dehiscence, incisional hernia. ${ }^{11}$ As the obesity alone may the important factor to determine the outcome of the surgical patients, this factor has not been studied or mentioned in our local literature.

\section{Methods}

This comparative study was conducted in three years from 01-05- 012 to30-04-2015 on 140 patients in the Department of Surgery of a public sector hospital. Both male and female patients of more than 12 years were included. The patients were divided into two groups of 70 patients each. The group A consisted of the obese and the group B consisted of nonobese patients. From their weight and height, the body mass index was calculated. The patients with diabetes mellitus, jaundice, peritonitis, renal failure, coagulopathy were excluded from the study. All the patients were routinely admitted for open and laparoscopic surgery on elective operation list .Clinical assessment and relevant investigations were done. They all were operated by the experienced consultant surgeon. The required operative procedure was performed and closed with polypropyelene in single, interrupted layer. They were managed postoperatively as per standard protocol. The operative and postoperative findings and outcome in term of wound complications (wound infection, seroma, dehiscence, incisional hernia) were noted .All these patients were followed for one year. The data was collected and analysed using SPSS version 20.

\section{Results}

In this study, 140 patients were included .All these patients were divided into two groups; group A (obese patients) and B (non obese patients) of 70 patients each. In group $A$, the age ranged from 17 to 58 years with mean of 37.10 (SD \pm 10.26$)$ years and males were common $(50.8 \%)$ than females $(49.2 \%)$ In group $\mathrm{B}$, the age ranged from 20 to 57 years with mean of 37.25 ( $\mathrm{SD} \pm 10.16$ ) years and males were common $(51.6 \%)$ than females $(48.4 \%)$. In group A, body mass index varied from $31.9 \mathrm{~kg} \mathrm{~m}^{-2}$ to $39.08 \mathrm{~kg} \mathrm{~m}^{-2}$ and in group B from $19.3 \mathrm{~kg} \mathrm{~m}^{-2}$ to $23.2 \mathrm{~kg} \mathrm{~m}^{-2}$. The surgical procedures performed are mentioned in Table 1. All these surgical wounds were clean or clean-contaminated. There was no opening or contamination by the bowel contents. Wound complications in both group were different in both groups (Table 2). These complications were wound infection, seroma, wound dehiscence/burst abdomen and incisional hernia. It was noticed that in group A patients, the complications were wound infection in $18(25.7 \%)$, seroma in 13 $(18.6 \%)$ dehiscence in $5(7.2 \%)$, and incisional hernia

Table 1: Surgical Procedures Performed.

\begin{tabular}{|c|l|c|c|}
\hline $\begin{array}{c}\text { Sr. } \\
\text { No. }\end{array}$ & Surgical Procedure & Group A & Group B \\
\hline 1 & Herniorrhaphy & 15 & 15 \\
\hline 2 & Hepatorrhaphy & 12 & 12 \\
\hline 3 & Appendicectomy & 14 & 14 \\
\hline 4 & Pelvic Mass excision & 5 & 7 \\
\hline 5 & Heller s Myotomy & 2 & 2 \\
\hline 6 & Cholecystectomy & 19 & 18 \\
\hline 7 & Nephrectomy & 3 & 2 \\
\hline
\end{tabular}


Table 2: Wound Complications.

\begin{tabular}{|c|l|l|l|}
\hline Sr. No. & Complications & No. Patients in Group A $(\mathbf{N}=\mathbf{7 0})$ & No. of Patients in Group B $(\mathbf{N}=\mathbf{7 0})$ \\
\hline 1. & Wound infection & $18(25.7 \%)$ & $3(4.3 \%)$ \\
\hline 2. & Seroma & $13(18.6 \%)$ & $5(7.2 \%)$ \\
\hline 3. & Wound dehiscence & $5(7.2 \%)$ & 00 \\
\hline 4. & Incisional hernia & $4(5.7 \%)$ & $1(1.4 \%)$ \\
\hline
\end{tabular}

$4(5.7 \%)$ patients. On the other hand, the complications in non obese (group B) were wound infection in 3 (4.3\%), seroma in $5(7.2 \%)$, hernia in $1(1.4 \%)$ and no dehiscence. The results showed that early and late wound complications are more in obese patients than nonobese patients. The Chi square test was used and there was significant association between obesity and wound complications ( $\mathrm{p}$-value $=0.072$ ).

\section{Discussion}

There are various morbid conditions which can affect the perioperative course in surgery. ${ }^{12}$ These problems need to be controlled for the better surgical outcome. Some of them (i.e. diabetes mellitus, hypertension) can be managed while others (i.e. obesity) are difficult or impossible to manage in perioperative period. ${ }^{13}$ The fat distribution is variable throughout the body compartments. Both the central and peripheral types of obesity carries increased risk of some diseases including diabetes mellitus, hypertension, arthritis, coronary heart disease and surgical complications. ${ }^{14}$

It is shown from the study that the wound complications occur relatively more commonly in patients with obesity. Obesity can affect the surgical outcome directly or indirectly being the cause of the systemic disorders. Even if the other systemic factors are excluded, obesity alone is important to determine the outcome of the surgical patients. ${ }^{15}$ The operative problems associated with obesity are difficulty in exposure and closure, port insertion in laparoscopic surgery, bleeding, prolonged operative time, visceral damage, wound infection, dehiscence, incisional hernia. ${ }^{16}$

The wound infection can be due type of surgery, diabetes mellitus, poor technique and immune disorders, obesity may also lead to this complication. If subcutaneous potential space is left during wound closure or the drain is not placed, seroma may form. ${ }^{17}$ The obese patients are more prone to develop seroma and wound infection despite all the precautions. ${ }^{18}$ Fat acts as pile deriver and the tensile strength of surgical wound is less in obesity. Difficulty during abdominal wound closure may also contribute towards early and late complications. Despite proper surgical technique, wound dehiscence and incisional hernia may occur. ${ }^{19}$

The study by Hourigan JS. et al, has shown that there has been increased recognition of the obesity regarding its contribution to worse outcomes in surgical patients. In particular, it has been up to $50 \%$ increased risk of wound infection among the obese patients undergoing abdominal surgery. This is more than our study and is because of the reason that they included patients with all types of the wounds. ${ }^{20}$

Giles KA. et al, described that obesity increases the wound infection and occasional mortality postoperatively. They conducted retrospective multi-institutional study to compare perioperative mortality and postoperative wound infection. Obese patients had a higher rate of wound infection than non-obese both after open and endovascular surgery They concluded that in obese patients, the risk of infectious complications was three times more than non obese after open and endovascular surgery. Whenever possible the obesity should be managed to avoid or minimize the perioperative complications. ${ }^{21}$ Although the decreased wound oxygen tension, impaired tissue antibiotic penetration and altered immune function have been mentioned for pathophysiology of the surgical site infection, the true effect of obesity has not been described.

Salani R. et al, have mentioned in their study on 72 cases with a body mass index of $\geq 40$ undergoing open abdominal surgery about the outcome of obese women in term of estimated blood loss, length of hospital stay, and postoperative wound complications. They have shown that 14 (20.9\%) cases had woundrelated infection. ${ }^{22}$ When compared with nonobese patients, morbidly obese patients also had a higher risk of pulmonary embolism, renal failure, dehiscence and incisional hernia. These results are similar to the wound infection in our study. 


\section{Conclusion}

The post-operative wound complications are significantly associated with obesity in patients undergoing abdominal surgery and are more in obese than nonobese patients.

\section{References}

1. Kassin MT, Owen RM, Perez SD, Leeds I, Cox JC, Schnier K, et al. Risk factors for 30-day hospital readmission among general surgery patients. Journal of the American College of Surgeons. 2012: 30215 (3): 322-30.

2. Adams KF, Leitzmann MF, Ballard-Barbash R, Albanes D, Harris TB, Hollenbeck A, et al. Body mass and weight change in adults in relation to mortality risk. American journal of epidemiology. 2014; 15179 (2): 135-44.

3. Shuster A, Patlas M, Pinthus JH, Mourtzakis M. The clinical importance of visceral adiposity: a critical review of methods for visceral adipose tissue analysis. The British journal of radiology. 2014.

4. Arroyo K, Herron DM. The epidemiology of obesity. InBariatric Endoscopy 2013 (pp. 1-9). Springer New York.

5. Di Saverio S. Emergency laparoscopy: a new emerging discipline for treating abdominal emergencies attempting to minimize costs and invasiveness and maximize outcomes and patients' comfort. Journal of Trauma and Acute Care Surgery. 2014; 177 (2): 338-50.

6. 6)Tuveri M, Tuveri A, Nicolo E, Tsuruma T, Nagayama M, Nakano S. et al. Incisional Hernia--" Difficult case" as specialistic case: real loss of substance, multi recurrences, infections, fistulas, lombocel, burst abdomen, reconstruction of the entire wall. Hernia. 2015; 119: S225.

7. Taylor A, DeBoard Z, Gauvin JM. Prevention of postoperative pulmonary complications. Surgical Clinics of North America. 2015; 3095 (2): 237-54.

8. Krezalek M, DeFazio J, Kim S, Khodarev N, Zaborin A, Christley S. et al. Program and Abstracts Thirty-fifth Annual Meeting of the Surgical Infection Society Westlake Village, California April 15-18, 2015. Surgical Infections. 2015; 116 (S1): S-1.

9. Adkinson JM, Garza III R, Shafqat MS, Miles MG. Considerations in Abdominal Wall Reconstruction. In Options in the Management of the Open Abdomen. 2015; (pp. 43-57). Springer New York.

10. Sandy-Hodgetts K, Carville K, Leslie GD. Determining risk factors for surgical wound dehiscence: a literature review. International wound journal. 2015; 112 (3): 265-75.

11. Banerjee A, Selzer DJ. Optimizing Perioperative Management: Perioperative Care and Protocols to Prevent and Detect Early Complications. In Bariatric Surgery Complications and Emergencies 2016; (pp. 31-49). Springer International Publishing.

12. Anderson DJ, Podgorny K, Berríos-Torres SI, Bratzler DW, Dellinger EP, Greene L. et al, Strategies to prevent surgical site infections in acute care hospitals: 2014 update. Infection Control \& Hospital Epidemiology. 2014; 1 35(06):605-27.

13. Woodcock SA. Perioperative Management of the Bariatric Surgery Patient. In Obesity, Bariatric and Metabolic Surgery 2016; (pp. 155-165). Springer International Publishing.

14. Aahlin EK, Tranø G, Johns N, Horn A, Søreide JA, Fearon KC. et al, Risk factors, complications and survival after upper abdominal surgery: a prospective cohort study. BMC surgery. 2015; 715 (1): 1.

15. Maggard-Gibbons M, Maglione M, Livhits M, Ewing B, Maher AR, Hu J, Li Z. et al, Bariatric surgery for weight loss and glycemic control in nonmorbidly obese adults with diabetes: a systematic review. Jama. 2013; 5309 (21): 2250-61.

16. Stranz C, Baker C, Singh S. Abdominal access techniques (including laparoscopic access). Surgery (Oxford). 2015; 3133 (5): 200-5.

17. Ho VP, Eachempati SR, Barie PS. Surgical Site Infections 11. Common Problems in Acute Care Surgery. 2013; 12: 139.

18. Gusenoff JA. Prevention and Management of Complications in Body Contouring Surgery. Clinics in plastic surgery. 2014; 3141 (4): 805-18.

19. Krpata DM, Blatnik JA, Novitsky YW, Rosen MJ. Evaluation of high-risk, comorbid patients undergoing open ventral hernia repair with synthetic mesh. Surgery. 2013; 31153 (1): 120-5.

20. Hourigan JS. Impact of obesity on surgical site infection in colon and rectal surgery. Clinics in colon and rectal surgery. 2011; 24 (04): 283-90.

21. Giles KA, Wyers MC, Pomposelli FB, Hamdan AD, Avery Ching Y, Schermerhom I. The impact of body mass index on perioperative outcomes of open and endovascular abdominal aortic aneurysm repair from the National Surgical Quality Improvement Program, 2005-2007.

22. Salani P. Gerardi MA, Harlin TN, Veras E, Bristow RE. Surgical approaches and short-term surgical outcomes for patients with morbid obesity and endometrial cancer. J Gynecol Surg. 2009; 25 (2): 41-S. 